\title{
Thin film evolution: Dewetting dynamics of a bimodal molecular system
}

\author{
J. M. Topple, ${ }^{*}$ S. A. Burke, S. Fostner, and P. Grütter \\ Department of Physics, McGill University, Montreal, Canada H3A 2T8
}

(Received 5 March 2009; published 18 May 2009)

\begin{abstract}
Deposition of submonolayer coverages of 3,4,9,10-perylenetetracarboxylic diimide onto $\mathrm{NaCl}(001)$ results in the bimodal growth of coexisting square and needle-shaped islands. The epitaxy of each island type was determined from molecular and atomic resolution noncontact atomic force microscopy images. Postdeposition, the needle islands grow while the square islands disappear completely within days. This is characterized as a dewetting transition, the dynamics of which has been described and empirically fit to the monomolecular growth model, a model typically applied to biological growth.
\end{abstract}

DOI: 10.1103/PhysRevB.79.205414

PACS number(s): 68.35.bm, 68.37.Ps, 68.43.Hn, 68.55.am

\section{INTRODUCTION}

The interesting optical and electronic properties of certain organic molecules have led to their use in a range of organic electronic and optoelectronic applications. ${ }^{1-3}$ To this end, thin film growth of a variety of molecules on different substrates has been studied. ${ }^{4,5}$ The morphology of thin films critically determines application-relevant properties, and can be controlled during growth to some degree by experimental parameters. ${ }^{2,6}$ However, postgrowth dynamics may change the film structure and resulting properties.

Bimodal growth systems exhibiting dewetting between two-dimensional (2D) and three-dimensional (3D) morphologies have been observed previously. ${ }^{6-11}$ The dewetting scenario is consistent for all bimodal and multimodal systems: atoms or molecules must overcome a potential energy barrier to reach the most stable state (the minimal free energy), and are limited by kinetic factors such as temperature and physical barriers (steps). This type of process, in general, is akin to other coarsening scenarios such as Ostwald ripening in that the growth process is competitive. Stable islands grow at the expense of metastable islands through mass transport driven by the minimization of the global free energy.

The analysis which applies to atomistic processes ${ }^{12-14}$ of this nature can also be applied to describe the dynamics of molecular dewetting. Thurmer et al. ${ }^{8}$ concluded that the transitional morphologies observed for $\mathrm{Ag}$ on $\mathrm{Si}(111)$ were influenced by substrate step density, annealing time, and temperature. These atomic systems are in many ways analogous to the molecular case. Bimodal growth has also been observed for molecular systems ${ }^{6,10}$ but the dynamics of the dewetting transition has thus far not been characterized. Kowarik et $a l .{ }^{15}$ concluded that organic materials have three additional issues which may alter growth behavior and epitaxy as compared to that for inorganic materials: (1) internal degrees of freedom (vibrational, conformational, and orientational), (2) molecule-molecule and molecule-substrate weak van der Waals force interactions, and (3) interaction potentials with surroundings spread over a large area. ${ }^{15}$

3,4,9,10-Perylenetetracarboxylic diimide (PTCDI) is an organic semiconductor with interesting electronic and optical properties $^{16-24}$ similar to the well-studied $3,4,9,10$ perylenetetracarboxylic dianhydride (PTCDA). ${ }^{25,26}$ The crys- talline structures of both molecules are influenced by hydrogen bonding between molecules. PTCDA anhydride groups tend to form hydrogen bonds to the perylene core edges while PTCDI N-H groups form hydrogen bonds to oxygen atoms of neighboring molecules, ${ }^{22}$ leading to the formation of different crystalline structures for the two very similar molecules.

The substrate also influences the growth of both PTCDA and PTCDI, ${ }^{16}$ and in the case of alkali halides the negative oxygen atoms are expected to sit over the substrate cations. ${ }^{6,23,27}$ Insulating substrates are an advantageous choice on which to build "proof of concept" molecular electronic devices, providing the ability to electrically isolate the device from the underlying surface. ${ }^{28}$ However, the surface science of molecules on insulators is not well understood, and therefore we attempt to obtain a more complete understanding of interactions between organic molecules and insulating substrates. Noncontact atomic force microscopy (NCAFM) is a nondestructive high-resolution technique ideal for imaging bulk insulator surfaces on which scanning tunneling microscopy is not possible. ${ }^{29,30} \mathrm{NC}$-AFM has been successfully used to image several systems of molecules deposited on insulators. ${ }^{31-33}$

The bimodal growth of PTCDI on $\mathrm{NaCl}$ is an interesting case in that one may observe in detail the dewetting transition over the course of days at room temperature. Other multimodal systems generally either dewet on much faster or slower time scales. Systems such as $\mathrm{C}_{60}$ on $\mathrm{NaCl}$ (Ref. 32) show evidence of dewetting but evolve too quickly to observe metastable structures at room temperature. Many other systems must be annealed to provide the additional energy to observe dewetting on a reasonable time scale. ${ }^{6-11}$

\section{EXPERIMENTAL PROCEDURES}

Preparation and characterization of samples took place in the JEOL JSPM 4500A ultrahigh-vacuum atomic force microscopy (AFM) system. Single-crystal NaCl (Korth Kristalle, Germany) samples were cleaved in situ and heated to $\sim 150{ }^{\circ} \mathrm{C}$ for $1 \mathrm{~h}$. A molecular evaporator (Kentax) was used to thermally deposit PTCDI (Alfa Aesar, 98\% purity) at $300{ }^{\circ} \mathrm{C}$ onto room-temperature substrates, having first been outgassed at $200{ }^{\circ} \mathrm{C}$ overnight. Deposition rates were calibrated using a quartz-crystal microbalance (Inficon) and 


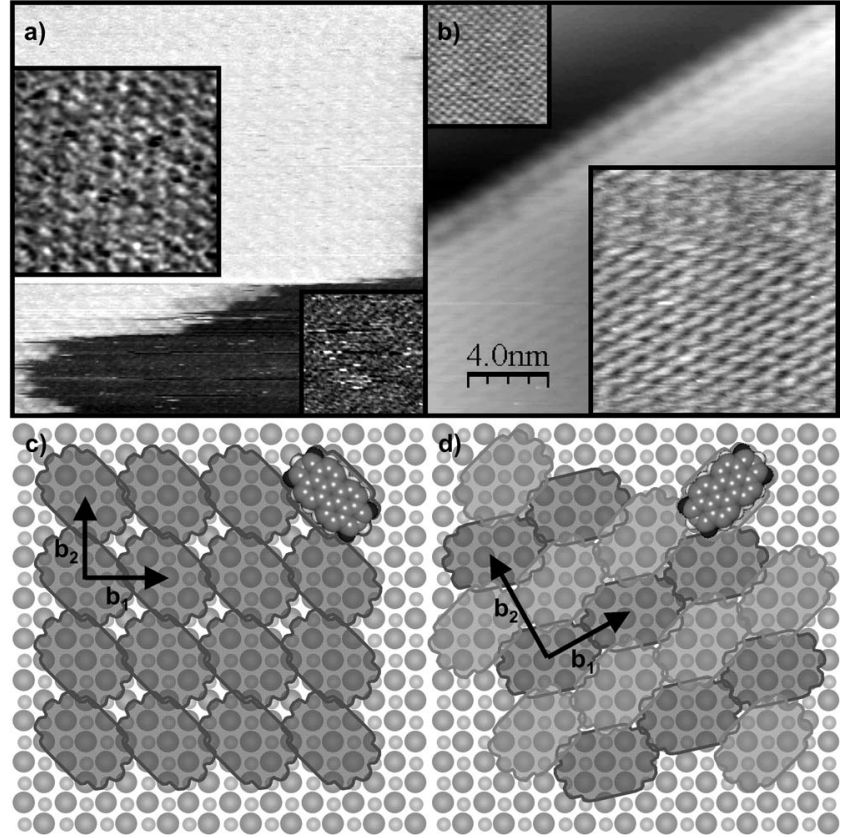

FIG. 1. [(a) and (c)] Molecular and atomic resolution images of (a) a $1 \mathrm{ML}$ square island of PTCDI on $\mathrm{NaCl}, \Delta f=-15 \mathrm{~Hz}$, and (b) a multilayer needle island of PTCDI on $\mathrm{NaCl}, \Delta f=-24 \mathrm{~Hz}$. Large and small inset regions of PTCDI and $\mathrm{NaCl}$, respectively, have been flattened and adjusted for contrast to make the structures more visible using WSXM (Ref. 34). (c) $2 \times 2$ brick wall square-island structure. (d) Needle-island structure (top layer of molecules only shown for clarity).

ranged from 0.008 to $0.015 \AA / \mathrm{s}$. Samples were subsequently imaged by NC-AFM using a modified Nanosurf easyPLL (phase-locked loop) with distance control for a constant frequency shift. Nanosensor cantilevers (PPP-NCLR) with typical resonance frequencies of $160-170 \mathrm{kHz}$, spring constants of $\sim 40 \mathrm{~N} / \mathrm{m}$, and oscillation amplitudes of 6-7 $\mathrm{nm}$ were used.

\section{RESULTS AND DISCUSSION}

\section{A. Epitaxy}

Images taken within a few hours of deposition reveal two coexisting morphologies: needle-shaped islands comprised of multiple layers of PTCDI with edges an angle of $16 \pm 1^{\circ}$ relative to the $\mathrm{NaCl}\langle 110\rangle$ directions and square-shaped 1-2 layer islands aligned with the $\langle 100\rangle$, shown in Fig. 2. The two shapes are associated with different epitaxies. Images with molecular resolution of the PTCDI and atomic resolution of the underlying $\mathrm{NaCl}$ were used to determine the structure of each island type, illustrated in Fig. 1.

\section{Square islands}

The square-shaped islands are usually comprised of a single layer of PTCDI with individual molecules oriented along the $\mathrm{NaCl}\langle 011\rangle$ directions. Based on the image in Fig. 1(a), we propose a $2 \times 2$ brick wall structure with each molecule in equivalent position with respect to the substrate, illustrated in Fig. 1(c). A stable second layer occasionally
TABLE I. Square and needle-island structure parameters.

\begin{tabular}{lcccc}
\hline \hline Structure & $\begin{array}{c}b_{1} \\
\AA\end{array}$ & $\begin{array}{c}b_{2} \\
(\AA)\end{array}$ & $\begin{array}{c}\theta \\
(\mathrm{deg})\end{array}$ & $\begin{array}{c}\beta \\
(\mathrm{deg})\end{array}$ \\
\hline Square & $11.3 \pm 0.4$ & $10.9 \pm 0.8$ & $1 \pm 3$ & $93 \pm 4$ \\
Needle & $13.2 \pm 0.3$ & $16.5 \pm 0.4$ & $29 \pm 1$ & $90 \pm 2$ \\
\hline \hline
\end{tabular}

forms provided the island area is large enough $\left(\gtrsim 400 \mathrm{~nm}^{2}\right)$ but is often observed to be transient. Molecules in square islands are highly mobile and therefore difficult to image. This indicates that they are less energetically favorable than needle islands, which sustain imaging frequency shifts on the order of $-20 \mathrm{~Hz}$ (normalized frequency shift of $-3 f N \sqrt{m})$ without alteration.

\section{Needle islands}

Needle-shaped islands have been observed exclusively with heights of two or more layers. The nucleation of three needle islands beginning with bilayers was captured in a sequence of NC-AFM images (see supporting information ${ }^{41}$ ), demonstrating a minimum of two layers required for the needle phase to exist. Needle islands nucleate preferentially at defects and step edges, a behavior described previously for stable $\mathrm{C}_{60}$ islands on $\mathrm{KBr}^{32}$ The crystalline structure of the needle islands resembles those determined for PTCDI on a variety of other substrates as well as the bulk $(10 \overline{2}) .{ }^{16-24}$ Lattice parameters determined from molecular and atomic resolution images for both needle and square islands are given in Table I.

\section{B. Dewetting}

At room temperature, the metastable square islands shrink and disappear within days of deposition while the stable needle islands grow larger, as illustrated in Fig. 2. Other images were taken well outside the consecutively imaged area to ensure that the NC-AFM tip did not influence the dewetting dynamics and indeed showed the same dewetting progress. Needle islands grow both in height and area, and tend to elongate rather than widen. This anisotropy is expected, given the hydrogen bonding that typically causes PTCDI molecules to form rows. The square islands of PTCDI on $\mathrm{NaCl}$ are comparable to the "metastable 2D islands" of $\mathrm{Au}$ observed on $\mathrm{SrTiO}_{3}(001)$ by Silly and Castell, ${ }^{9}$ the "transitional morphologies" of $\mathrm{Ag}$ on $\mathrm{Si}(111)$ described by Thurmer et al., ${ }^{8}$ as well as the metastable monolayer (ML) $p 3 \times 3$ structure of PTCDA molecules on $\mathrm{NaCl}$ reported by Burke et al., ${ }^{6}$ all of which dewet upon annealing.

\section{Evolution of island populations}

The population of molecules in each island type was calculated from the measured volumes and density calculated from the lattice constants determined above and a measured needle interlayer spacing of $0.315 \pm 0.015 \mathrm{~nm}$ (assuming the same interlayer spacing for the square structure, a reasonable assumption for perylenes ${ }^{17}$ ), 

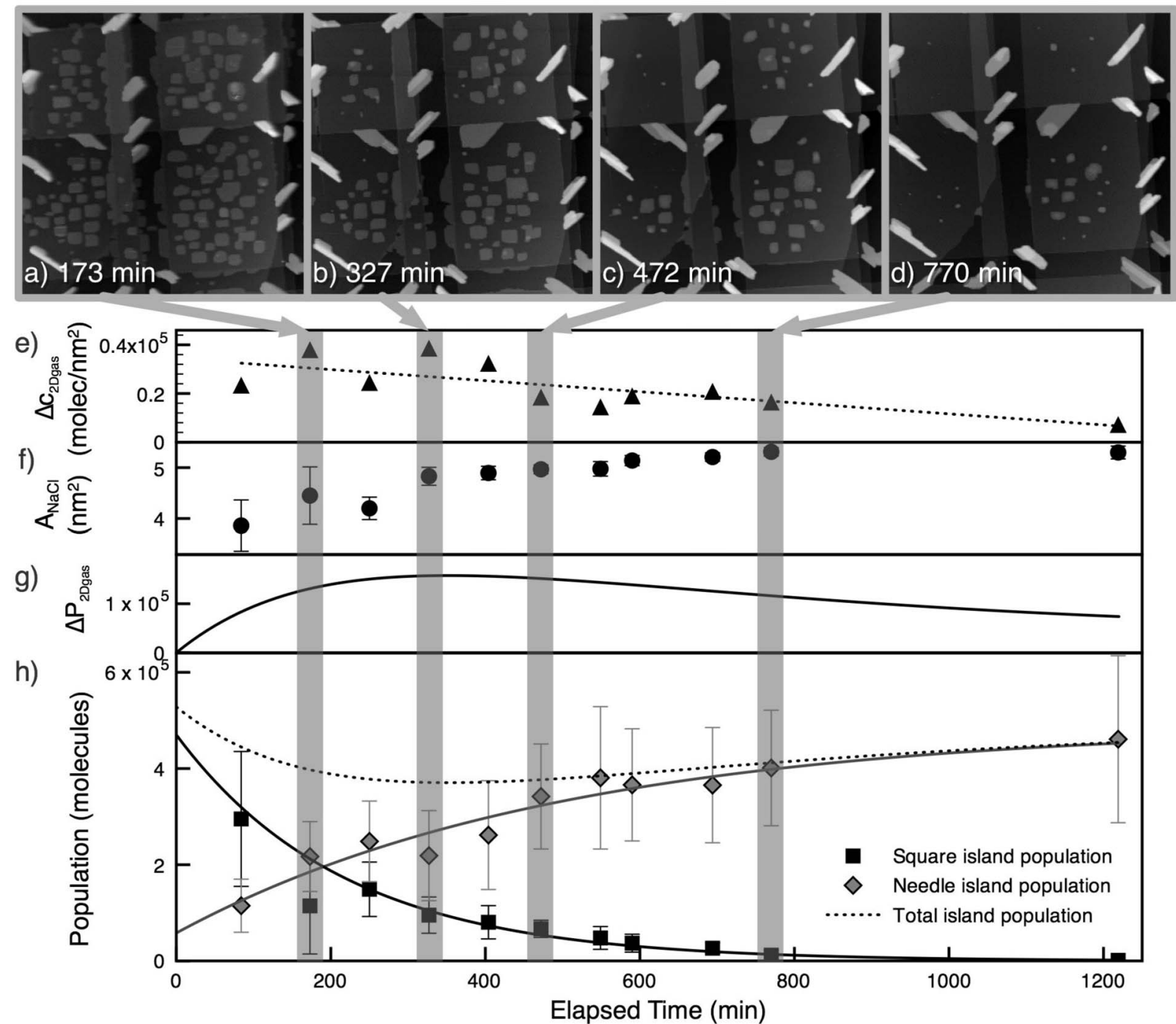

FIG. 2. Postdeposition growth of needle islands and the disappearance of square islands over time. [(a)-(d)] $770 \times 770 \mathrm{~nm}^{2}, \Delta f=-2-$ $-4 \mathrm{~Hz}, 0.3 \mathrm{ML}$ PTCDI on $\mathrm{NaCl}$ images, and corresponding time since deposition, with both square and needle-shaped islands visible. (e) Differential concentration of 2D molecular gas (molecules $/ \mathrm{nm}^{2}$ ), (f) area of exposed $\mathrm{NaCl}$ substrate, (g) differential population of $2 \mathrm{D}$ molecular gas (population less an unknown steady-state offset), and (h) population of square and needle islands plotted as functions of time, with monomolecular growth and decay fits.

$$
P_{\text {isltype }}=\left(\sum A_{\text {isl }}\right) \bar{h}_{\text {isl }} n_{\text {molec }}
$$

where $P_{\text {isltype }}$ is the number of molecules in each island type per image, $A_{\text {isl }}$ is the area of each island, $\bar{h}_{\text {isl }}$ is the average island height, and $n_{\text {molec }}$ is the molecule number density. The resulting populations were plotted as functions of time and fit as shown in Fig. 2(h). Error bars represent one standard deviation of population per island type per image, and are large in the needle-island case due to variations in needle-island height (some islands straddle multiple terraces and/or have incomplete upper layers).

An empirical fit was used to quantify the time scale of the dewetting transition. The growth function found to best fit the data for the needle (stable) islands is that of monomolecular growth, which is generally used to describe growth of biological systems. ${ }^{35}$ This function assumes that the growth rate of a particle (island) is proportional to the difference between the current and final sizes which is consistent with a finite number of units (molecules) in the system. As the particle size increases the growth rate slows due to the reduction in available resources from the background concentration field,

$$
\frac{d P}{d t}=\frac{1}{\tau}\left(P_{\infty}-P\right),
$$

where $P$ is the current population of molecules (size of the island), $\tau$ is a time constant $\tau>0$, and $P_{\infty}$ is the final population of molecules in the island. Considering the stable islands as sinks for the background concentration field, the right hand term can also be considered a flow of molecules into the island: the change in island size corresponds to the number of molecules that arrive and attach in a characteristic 
TABLE II. Fit parameters for monomolecular growth/decay of needle/square islands.

\begin{tabular}{lcc}
\hline \hline Island type & Needle & Square \\
\hline$P_{\infty}\left(\times 10^{5}\right)$ & $50 \pm 9$ & 0 \\
$P_{o}\left(\times 10^{5}\right)$ & $6 \pm 7$ & $48 \pm 8$ \\
$\tau(\min )$ & $500 \pm 200$ & $220 \pm 10$ \\
\hline \hline
\end{tabular}

time. In this way, the time constant $\tau$ encapsulates both diffusion and attachment parameters. This solves to the growth function:

$$
P=P_{\infty}-\delta e^{-t / \tau},
$$

where $\delta$ is the difference between the initial and final populations $\left(P_{\infty}-P_{o}\right)$.

An analogous decay function was fit to the square-island population,

$$
P=P_{o} e^{-t / \tau},
$$

which similarly illustrates the flow of molecules out of these islands.

The fit parameters for both island types are given in Table II. $P_{o}$ and $P_{\infty}$ are the initial and postdewetting island populations but the time constant $\tau$ provides some quantitative insights into the dynamics of dewetting and could be used for comparison with modeling. $\tau_{\text {Needle }}$ and $\tau_{\text {Square }}$ reflect the net capture rate of molecules into the needle islands and the net escape rate out of the square islands, respectively. The diffusion constant, coverage, step density, geometry, and sticking probability for different island edges are all factors which will affect $\tau$. The rate of dewetting is expected to be tunable by adjusting experimental parameters such as temperature ${ }^{6}$ and substrate preparation to induce desired film behavior. An independent data set with significantly lower step density and 0.2 ML coverage was observed to dewet with just over double the above $\tau$ values (see supporting information $^{41}$ ) and produced considerably larger islands, demonstrating the combined influence of substrate structure and coverage. Due to the predominant nucleation of needle islands at step edges, the dewetting process is dependent on the initial state of the sample, and is highly sensitive to substrate structure. Substrate areas with high step density tend to contain many small needle islands and dewet quickly while areas with low step density tend to slowly form fewer larger islands as dewetting is limited by diffusion and nucleationsite density. The influence of these parameters on the time scale for dewetting is a subject of future interest.

The observed dynamical behavior of PTCDI dewetting is similar to that recently reported by Käfer et al. ${ }^{36}$ for pentacene on $\mathrm{SiO}_{2}$ and gold. The transition from the "thin film phase" of pentacene to a bulklike phase was monitored dynamically by $\mathrm{x}$-ray photoelectron spectroscopy measurements of the covered and bare surface areas. The surface area covered with pentacene, i.e., the total integrated base of all islands, was found to fit well to a sigmoidal Weibull function which transitions from the initial covered area to the final covered area exponentially. Although the time constants found in that study differ by approximately an order of magnitude, likely a result of faster diffusion of pentacene on $\mathrm{SiO}_{2}$ and gold than the larger PTCDI on $\mathrm{NaCl}$, the dynamical behavior is strikingly similar.

\section{2D Molecular gas}

If the flux of molecules out of the square islands equals the flux into the needle islands (steady state), then $\tau$ should be the same for both fits and they should sum to a constant (assuming negligible desorption at room temperature). This is not the case (as verified by an independent data set; see supporting information $\left.{ }^{41}\right)$. Since $\tau$ is shorter for the square islands and, as they dewet, additional $\mathrm{NaCl}$ surface area is exposed, we propose that the lost population of molecules go into another state, diffusing over the surface in the form of a 2D molecular gas ${ }^{37}$ faster than the AFM is able to image.

One must now consider three fluxes: the capture rate for square islands (which becomes negative after deposition), the capture rate for needle islands, and the flux in and out of the $2 \mathrm{D}$ gas. The sum of the three populations of molecules can be written as

$$
P_{\text {total }}=P_{\text {needle }}+P_{\text {square }}+P_{2 \mathrm{D} \mathrm{gas}} .
$$

To account for the initial decrease and subsequent increase in total island population, consider that

$$
P_{2 \mathrm{D} \mathrm{gas}}=c_{2 \mathrm{D} \mathrm{gas}} A_{\mathrm{NaCl}},
$$

where $c_{2 \mathrm{D} \text { gas }}$ is the $2 \mathrm{D}$ gas number density or concentration and $A_{\mathrm{NaCl}}$ is the area of exposed $\mathrm{NaCl}$ substrate. This neglects the population of molecules diffusing on the islands (which should be reduced given the energy cost of interlayer mass transport $\left.{ }^{12,38}\right)$.

The time evolution of the $2 \mathrm{D}$ gas population is shown in Fig. 2(g). Taking the measured differential population of the 2D gas and exposed area of the $\mathrm{NaCl}$ [see Fig. 2(f)] as the area in which this population resides, the differential $2 \mathrm{D}$ concentration can be calculated as a function of time [see Fig. 2(e) ]. An undetermined offset may also be present. The decreasing number density of the $2 \mathrm{D}$ gas reflects a concentration field which is not constant with time but rather becomes depleted as molecules are incorporated into the stable needleisland population and fewer molecules are supplied by the square islands. $P_{2 \mathrm{D} \text { gas }}$ initially increases as more $A_{\mathrm{NaCl}}$ is exposed by dewetting then decreases with $c_{2 \mathrm{D} \text { gas }}$ as $A_{\mathrm{NaCl}}$ approaches a constant [see Fig. $2(\mathrm{~g})]$. This differs with other models of ripening behavior which usually consider a constant concentration field. This is also consistent with the observation that no significant further ripening of the needle islands is observed after the total consumption of the metastable island population.

\section{Evolution of island size distribution}

The use of a scanning probe technique also allows for an analysis of the size distribution of the islands and how these evolve with time. Figure 3 shows the island volume distributions of the square and needle islands at different times. The square islands decrease in both size and number, as can be seen from the histograms which shift toward smaller island 
(a)

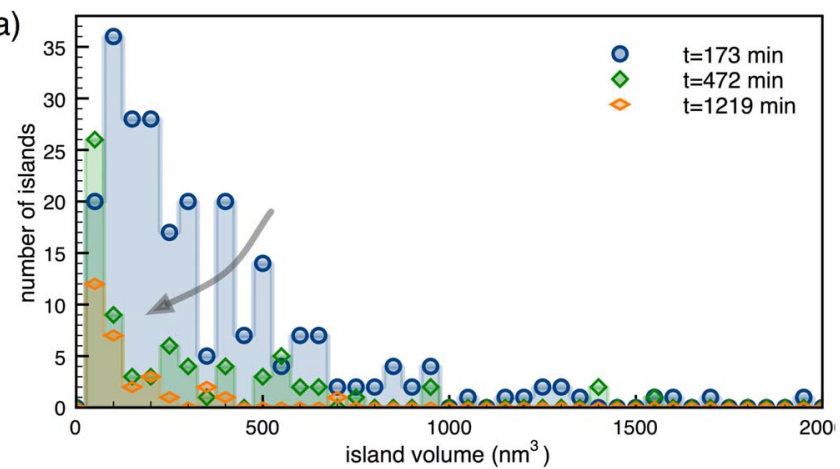

(b)

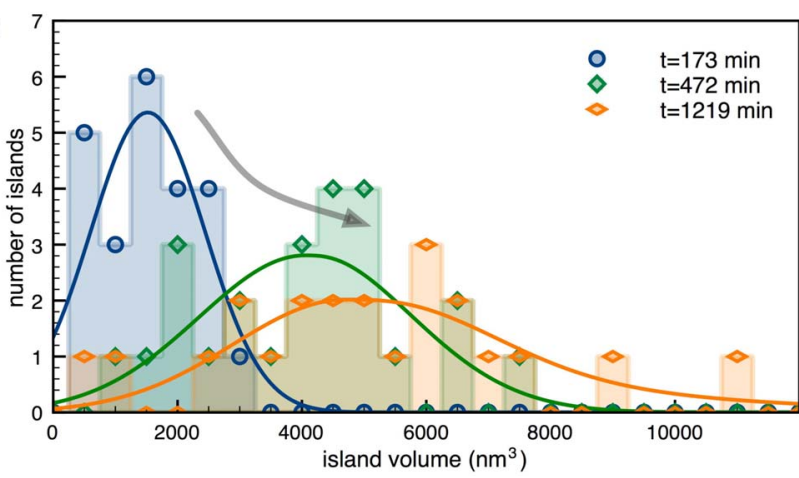

FIG. 3. (Color online) Island volume histograms of (a) square (metastable) islands and (b) needle (stable) islands for three different times. Solid lines in (b) are fits to a Gaussian with an exponential tail and are intended as a guide to the eye.

volume. The needle islands (in the pure-growth regime) both grow in size and have increasingly broad distributions. At longer times, the needle islands also appear to exhibit a large size tail which is atypical of processes such as Ostwald ripening.

Several factors may influence the observed sizedistribution evolution. In typical coarsening scenarios, the background concentration field is assumed (a) constant and (b) homogeneous. As shown for the dewetting process observed here, the concentration field feeding the growth of stable needle islands decreases with time, limiting the growth process. The needle islands act as sinks (with higher stability and higher sticking probability) and the square islands act as sources (with lower stability and therefore higher molecule escape probability). Close inspection of the images reveals that the square islands closest to the needle islands dewet faster than those farther away, resulting in clusters of square islands of which the edge islands shrink and disappear first, creating depletion regions. If the cluster of square islands is large, edge islands may also appear to retreat from the needle islands (see supporting information ${ }^{41}$ ). Shrinking indicates that the net flux of molecules out of a square island is greater than the flux in, and retreating indicates that the flux of molecules in and out of an island is not isotropic. Since all sides of the square islands are crystallographically identical, a difference in flux indicates a difference in the local 2D gas number density. In other words, this indicates that the $2 \mathrm{D}$ gas number density is lower near the growing needle islands and higher among clusters of the metastable square islands. Under this interpretation, the steady-state 2D gas number density required to maintain a constant square island population (independent of any Ostwald ripening which might occur within this population) would be higher than that for a constant needle island population; hence the bimodal system dewets. The difference in time scales between the disappearance of square (metastable) and growth of needle (stable) islands supports the supposition that the background concentration field is inhomogeneous.

We also observe that the needle islands do not grow isotropically but rather have different growth rates in each dimension, growing fastest in length: $d l / d t>d h / d t, d w / d t$. An added complication in analyzing the size distributions of the needle islands arises from the tendency for these islands to nucleate at steps or other defects. Ideally, the dewetting process would be studied on a perfect flat crystalline terrace so that the homogeneous growth of the needle islands would not be influenced by adjacent islands. However, due to the surprisingly large diffusion length observed for PTCDI on $\mathrm{NaCl}$, as with other molecules on alkali halides, ${ }^{6,32}$ needle islands are always heterogeneously distributed at step edges or other defects, which act as traps ${ }^{13}$ and may result in nonnegligible spatial correlations between neighboring islands. For example, adjacent needle islands in similar orientations occasionally coalesce but growth toward a perpendicular neighboring island has been observed to cease before coalescence can occur (see supporting information ${ }^{41}$ ).

\section{CONCLUSION}

Bimodal growth and postdeposition dewetting of PTCDI on $\mathrm{NaCl}$ have been observed. Molecules diffuse out of metastable square islands to join more energetically favorable needle islands over time. We find that the dynamics of this transition is empirically well described by the monomolecular growth model, which is anticipated to be applicable to bimodal systems, in general, although the dewetting time scale may vary significantly. The dynamics of dewetting quantified here is expected to be tunable via experimental parameters (such as temperature, substrate preparation, etc.), which can also impact the resulting growth structures. The characterization of the influence of these factors is a subject for continued exploration. Comparative modeling, such as within a kinetic Monte Carlo framework, ${ }^{39,40}$ is required to discern the effect of kinetic factors and individual molecular scale processes on the overall growth dynamics, for which this quantitative analysis provides a basis. The characterization and understanding of this dynamical evolution is an important step in controlling the structure of thin organic films for nanoscale devices which are sensitive to the nanoscale film structure.

\section{ACKNOWLEDGMENTS}

The authors wish to thank Martin Grant for a helpful discussion of Ostwald ripening, and the following funding agencies: NSERC, CFI, FQRNT, and CIfAR. 
*Author to whom correspondence should be addressed; topplej@physics.mcgill.ca

${ }^{1}$ C. D. Dimitrakopoulos and P. R. L. Malenfant, Adv. Mater. 14, 99 (2002).

${ }^{2}$ G. Horowitz, Adv. Mater. 10, 365 (1998).

${ }^{3}$ S. R. Forrest, Nature (London) 428, 911 (2004).

${ }^{4}$ G. Witte and C. Woll, J. Mater. Res. 19, 1889 (2004).

${ }^{5}$ D. E. Hooks, T. Fritz, and M. D. Ward, Adv. Mater. 13, 227 (2001).

${ }^{6}$ S. A. Burke, W. Ji, J. M. Mativetsky, J. M. Topple, S. Fostner, H. J. Gao, H. Guo, and P. Grutter, Phys. Rev. Lett. 100, 186104 (2008).

${ }^{7}$ K. J. Wan, X. F. Lin, and J. Nogami, Phys. Rev. B 47, 13700 (1993).

${ }^{8}$ K. Thurmer, E. D. Williams, and J. E. Reutt-Robey, Phys. Rev. B 68, 155423 (2003).

${ }^{9}$ F. Silly and M. R. Castell, Phys. Rev. Lett. 96, 086104 (2006).

${ }^{10}$ T. Dienel, C. Loppacher, S. C. B. Mannsfeld, R. Forker, and T. Fritz, Adv. Mater. 20, 959 (2008).

${ }^{11}$ B. Krause, A. C. Dürr, F. Schreiber, and H. Dosch, J. Chem. Phys. 119, 3429 (2003).

${ }^{12}$ Z. Y. Zhang and M. G. Lagally, Science 276, 377 (1997).

${ }^{13}$ J. A. Venables, Introduction to Surface and Thin Film Processes (Cambridge University Press, Cambridge, England, 2000).

${ }^{14}$ W. K. Burton, N. Cabrera, and F. C. Frank, Philos. Trans. R. Soc. London, Ser. A 243, 299 (1951).

${ }^{15}$ S. Kowarik, A. Gerlach, and F. Schreiber, J. Phys.: Condens. Matter 20, 184005 (2008).

${ }^{16}$ C. Ludwig, B. Gompf, J. Petersen, R. Strohmaier, and W. Eisenmenger, Z. Phys. B: Condens. Matter 93, 365 (1994).

${ }^{17}$ P. M. Kazmaier and R. Hoffmann, J. Am. Chem. Soc. 116, 9684 (1994).

${ }^{18} \mathrm{G}$. Klebe, data deposited at the Cambridge Crystallographic Data center (CCDC), File CIF_LENPEZ, CSD No. 55462 (1994).

${ }^{19}$ B. Uder, C. Ludwig, J. Petersen, B. Gompf, and W. Eisenmenger, Z. Phys. B: Condens. Matter 97, 389 (1995).

${ }^{20}$ K. Tojo and J. Mizuguchi, Z. Kristallogr. 217, 253 (2002).

${ }^{21}$ O. Guillermet, A. Glachant, J. Y. Hoarau, J. C. Mossoyan, and M. Mossoyan, Surf. Sci. 548, 129 (2004).

${ }^{22}$ J. C. Swarbrick, J. Ma, J. A. Theobald, N. S. Oxtoby, J. N. O'Shea, N. R. Champness, and P. H. Beton, J. Phys. Chem. B 109, 12167 (2005).
${ }^{23}$ T. Kunstmann, A. Schlarb, M. Fendrich, T. Wagner, R. Moller, and R. Hoffmann, Phys. Rev. B 71, 121403 (2005).

${ }^{24}$ O. Guillermet, A. Glachant, M. Mossoyan, and J. C. Mossoyan, J. Phys. IV 132, 77 (2006).

${ }^{25}$ Y. Hirose, A. Kahn, V. Aristov, P. Soukiassian, V. Bulovic, and S. R. Forrest, Phys. Rev. B 54, 13748 (1996).

${ }^{26}$ S. Antohe, N. Tomozeiu, and S. Gogonea, Phys. Status Solidi A 125, 397 (1991).

${ }^{27}$ M. Mobus, N. Karl, and T. Kobayashi, J. Cryst. Growth 116, 495 (1992).

${ }^{28}$ W. Ji, S. A. Burke, H.-J. Gao, P. Grutter, and H. Guo (unpublished).

${ }^{29}$ T. R. Albrecht, P. Grutter, D. Horne, and D. Rugar, J. Appl. Phys. 69, 668 (1991).

${ }^{30} \mathrm{~S}$. Morita, R. Wiesendanger, and E. Meyer, Noncontact Atomic Force Microscopy, Nanoscience and Technology (Springer, New York, 2002).

${ }^{31}$ T. Fukuma, K. Kobayashi, K. Noda, K. Ishida, T. Horiuchi, H. Yamada, and K. Matsushige, Surf. Sci. 516, 103 (2002).

${ }^{32}$ S. A. Burke, J. M. Mativetsky, R. Hoffmann, and P. Grutter, Phys. Rev. Lett. 94, 096102 (2005).

${ }^{33}$ J. M. Mativetsky, S. A. Burke, S. Fostner, and P. Grutter, Nanotechnology 18, 105303 (2007).

${ }^{34}$ I. Horcas, R. Fernandez, J. M. Gomez-Rodriguez, J. Colchero, J. Gomez-Herrero, and A. M. Baro, Rev. Sci. Instrum. 78, 013705 (2007).

${ }^{35}$ A. S. Karkach, Demogr. Res. 15, 347 (2006).

${ }^{36}$ D. Käfer, C. Wöll, and G. Witte, Appl. Phys. A: Mater. Sci. Process. 95, 273 (2009).

${ }^{37}$ S. Berner, M. Brunner, L. Ramoino, H. Suzuki, H. J. Guntherodt, and T. A. Jung, Chem. Phys. Lett. 348, 175 (2001).

${ }^{38}$ D. Y. Zhong, M. Hirtz, W. C. Wang, R. F. Dou, L. F. Chi, and H. Fuchs, Phys. Rev. B 77, 113404 (2008).

${ }^{39}$ D. Choudhary, P. Clancy, R. Shetty, and F. Escobedo, Adv. Funct. Mater. 16, 1768 (2006).

${ }^{40}$ R. Ruiz, D. Choudhary, B. Nickel, T. Toccoli, K. Chang, A. C. Mayer, P. Clancy, J. M. Blakely, R. L. Headrick, S. Iannotta, and G. Malliaras, Chem. Mater. 16, 4497 (2004).

${ }^{41}$ See EPAPS Document No. E-PRBMDO-79-038920 for movie files of time lapsed dewetting. For more information on EPAPS, see http://www.aip.org/pubservs/epaps.html. 